\title{
A Stimuli-Responsive Double-Stranded Digold(I) Helicate
}

\author{
Csaba Jobbágy, ${ }^{a}$ Miklós Molnár, ${ }^{a}$ Péter Baranyai, ${ }^{b}$ Andrea Hamza, ${ }^{c}$ Gábor Pálinkás ${ }^{a}$ and Andrea Deák ${ }^{\star a}$
}

CRYSTENGCOMM (ISSN: 1466-8033) 16: pp. 3192-3202. (2014)

\section{DOI:10.1039/C3CE42474J}

${ }_{5}$ We report herein a stimuli-responsive dinuclear double stranded $\left[\mathrm{Au}_{2} \mathrm{~L}_{2}\right]^{2+}$ helicate assembled from gold(I) atoms and phenyl-substituted diphosphine ligands derived from xanthene-type backbone $(\mathrm{L})$. The conformational flexibility of the dinuclear $\left[\mathrm{Au}_{2} \mathrm{~L}_{2}\right]^{2+}$ helicate allows a diversity of 10 molecular conformations and packing arrangements that lead to different solid-state emission colours. Blue $\left(\mathrm{I}_{\mathrm{B}}\right)$, bluish green $\left(\mathrm{II}_{\mathrm{G}}\right)$ and yellow ( $\left.\mathrm{III}_{\mathrm{Y}}\right)$ emitting crystalline and red emitting $\left(I V_{R}\right)$ amorphous forms of this double stranded $\left[\mathrm{Au}_{2} \mathrm{~L}_{2}\right]^{2+}$ helicate have been obtained by slight modification 15 of the crystallization conditions. Different molecular conformations, packing arrangements of dinuclear double stranded $\left[\mathrm{Au}_{2} \mathrm{~L}_{2}\right]^{2+}$ helicates that result in different noncovalent interactions have played the most significant role in tailoring the solid-state luminescent properties. On the basis 20 of the single crystal structural data and photophysical studies, we found that increasing number of intra- and mainly intermolecular noncovalent interactions lock and rigidify the twisted conformation of the double stranded $\left[\mathrm{Au}_{2} \mathrm{~L}_{2}\right]^{2+}$ helicate and enhanced $\pi$-stacking between its aromatic units ${ }_{25}$ induced the red-shift in solid-state luminescence emission. The solid-state luminescence colour of this double stranded $\left[\mathrm{Au}_{2} \mathrm{~L}_{2}\right]^{2+}$ helicate can be switched reversibly from blue to red by external (mechanical and chemical) stimuli.

\section{INTRODUCTION}

30 The understanding and manipulation of the light emitting behaviour of solid-state emitting luminescent materials is a topic of current research interest. ${ }^{1-8}$ Modification or alteration of molecular structures and/or packing arrangements is the most common approach for tuning and controlling the emissions of 35 luminescent compounds in the solid state. One approach to achieve various emission colours from the same compound is the existence of different crystalline and amorphous phases displaying different molecular conformations or packings. ${ }^{6-8}$ Generally, the molecular packing is controlled by subtle interplay 40 between different noncovalent intermolecular interactions among the components, such as cations, anions, solvents, etc. Therefore, variations in noncovalent intermolecular interactions between the building blocks of a crystal could be an effective strategy to finetune its solid-state luminescent properties. ${ }^{8}$ Another strategy

45 involves the controlling of molecular structures and/or packings with external stimuli such as thermal, optical, electrical, mechanical, solvent vapour etc. leads to the development of stimuli-responsive luminescent materials whose emissions can be repeatedly switched between different emission colours. ${ }^{1-8}$ ${ }_{50}$ Mechanochromic luminescent materials dynamically switch their luminescence colour by responding to mechanical stimuli such as grinding, pressing or shearing. ${ }^{1-5}$ The subsequent reversion to their original luminescence colour is usually achieved upon recrystallization or thermal treatment. ${ }^{1-5,9-11}$ These materials have 55 attracted increased attention because of their potential applications in optical storage and memories, mechanical sensors, displacement and deformation detectors, optoelectronic and display devices. ${ }^{1}$ Such materials could also serve as models for studying the luminescent colour changes associated with 60 structural changes of their molecular assemblies that switch from one state to another under mechanical stimuli. However, despite their importance in future technology development the design principles of mechanochromic luminescent materials is less well studied. ${ }^{12-14}$ Further in-depth understanding of the underlying 65 molecular mechanism may provide the basis for improved and innovative design strategies for novel mechanochromic luminescent materials. Very recently it was reported that some aggregation-induced emission (AIE) active organic compounds also exhibit mechanochromic luminescent properties. $^{12-21}$ 70 Molecules with AIE activity emit more efficiently in solid-state (nanoparticle, powder, crystal, etc.) than in the dissolved form. ${ }^{15-}$

${ }^{21}$ However, it was suggested that AIE active organic molecules with propeller-like conformation bearing aromatic rotors might be ideal candidates as mechanochromic materials. ${ }^{12-14}$ The 75 multiple phenyl functions of these molecules undergo active intramolecular rotations, which quench their emission in solution. The dynamic intramolecular motions, e.g. rotation of phenyl functions, are restricted in the solid-state rendering the molecules emissive. ${ }^{18,19}$ This AIE phenomenon is opposite to commonly 80 observed aggregation-caused quenching. ${ }^{18,19}$ However, there are still no examples of metal complexes that simultaneously exhibit AIE activity, phase-dependent and stimuli-responsive luminescent properties. Therefore, it is not astonishing that the general design principles of AIE active mechanochromic 85 luminescent metal compounds, particularly those with remarkably tuneable emission wavelength can not be found in the literature.

Most of gold(I) complexes are luminescent, and with a judicious choice of ligands or anions, coordination geometry, and 90 the extent of aurophilic $\mathrm{Au} \cdots \mathrm{Au}$ interaction it is possible to tune the emission energies of these compounds over a wide spectral range. ${ }^{22-24}$ In some cases different luminescent colours of gold(I) complexes emerge as a result of the formation of crystalline 
polymorphs. ${ }^{25-31}$ There are just some examples, in which gold(I) complexes were found to crystallize concomitantly. ${ }^{25-27}$

The luminescence from gold(I) complexes has also attracted considerable attention because of the range of intriguing 5 luminescence thermochromism, mechanochromism, vapochromism as well as solvoluminescent properties that have resulted in response to various external stimuli. ${ }^{32-41}$ There are, however, a relatively limited number of mechanochromic luminescent gold complexes reported in the literature. ${ }^{36-44}$ Balch 10 reviewed such visually detected changes in the luminescence of gold and other transition metal complexes. ${ }^{2}$ Fackler and coworkers reported that the grinding of the non-luminescent crystals of $\quad\left[(\mathrm{TPA})_{2} \mathrm{Au}\right][\mathrm{AuCN}] \quad(\mathrm{TPA}=$ 1,3,5-triaza-7-phosphaadamantane) initiates a strong green emission. ${ }^{36}$ Eisenberg and 15 co-workers have found that a weakly emissive thiouracil (tuH) gold(I) complex, $\left[\mathrm{Au}_{2}(\mu\right.$-tuH $\left.)(\mu-\mathrm{dppm})\right]\left(\mathrm{CF}_{3} \mathrm{CO}_{2}\right)$ after gentle crushing exhibits intense blue luminescence as a result of a molecular structure modification occurred upon a solid- state chemical reaction (release of $\mathrm{CF}_{3} \mathrm{CO}_{2} \mathrm{H}$ ). ${ }^{40}$ However, up to now, 20 only two gold(I) complexes that exhibit reversible mechanochromic luminescence have been reported. ${ }^{42,43}$ Ito and Sawamura reported the $\left[\left(\mathrm{F}_{5} \mathrm{C}_{6} \mathrm{Au}\right)_{2}\left(\mu-1,4-\mathrm{CNC}_{6} \mathrm{H}_{4}\right)\right]$ complex that shows a change in its luminescence colour from blue to yellowgreen upon grinding and subsequent reversion to its original 25 emission colour upon solvent treatment. ${ }^{42}$ The intense blue luminescence of $\left[\mathrm{Au}(\mathrm{dppb})_{2}\right] \mathrm{BF}_{4}$ complex $(\mathrm{dppb}=1,2-$ bis(diphenylphosphino) benzene) after continuous grinding changed into yellow orange and its original emission recovered in the presence of VOCs. ${ }^{43}$

30 Helicates formed by spontaneous and cooperative selfassembly of metal ions and ligand strands attracted much attention from the scientific community as helicity is an essential element of life and is also important in functional materials. ${ }^{45}$ Dinuclear double-stranded helicates consisting of gold(I) atoms 35 and diphosphine ligands (diphos) have been reported by us and others. $^{46-49,50}$ These helicates whose axis consists of short $\mathrm{Au} \cdots \mathrm{Au}$ contacts show dynamic switching between enantiomers in solution. ${ }^{46-48,50}$

On the basis of above considerations, dinuclear double40 stranded helicate constructed from luminescent gold(I) atoms and diphosphine ligands bearing aromatic rotors are of particular interest for in-depth investigation and potential application as consequence of having conformationally flexible core and wellsuited luminescent properties. As a result of conformationally 45 flexibility, it is rational to envisage that several solid-state forms of dinuclear double-stranded helicate could be obtained by the choice of crystallization conditions (solvent, concentration, temperature, etc.). However, different molecular conformations and packing arrangements could play significant role in tailoring 50 the solid-state luminescent properties. Moreover, we envisage that these helicates could also exhibit stimuli responsive properties. However, it is apparent that effective control of any functional system rests upon the comprehensive understanding of the origin of the stimuli-responsive behaviour.

\section{${ }_{55}$ RESULTS AND DISCUSSION}

\section{Double Stranded Digold(I) Helicate with Tuneable Luminescent Properties}

Earlier we reported the synthesis of a $\left[\mathrm{Au}_{2} \mathrm{~L}_{2}\right]\left(\mathrm{NO}_{3}\right)_{2}$ (1) complex consisting of dinuclear double stranded $\left[\mathrm{Au}_{2} \mathrm{~L}_{2}\right]^{2+}$ ${ }_{60}$ helicate assembled from gold(I) cations and phenyl-substituted diphosphine ligands derived from rigid xanthene-type backbone, namely 9,9-dimethyl-4,5-bis(diphenylphosphino)-xanthene (L). ${ }^{46}$ This double stranded $\left[\mathrm{Au}_{2} \mathrm{~L}_{2}\right]^{2+}$ helicate bearing aromatic rotors exists in a twisted figure-eight conformation in the crystalline ${ }_{65}$ state. ${ }^{46}$ However, dynamic interconversion between figure-eight conformational enantiomers of double stranded $\left[\mathrm{Au}_{2} \mathrm{~L}_{2}\right]^{2+}$ helicate (Fig. 1) occurred in solution. ${ }^{46,47}$

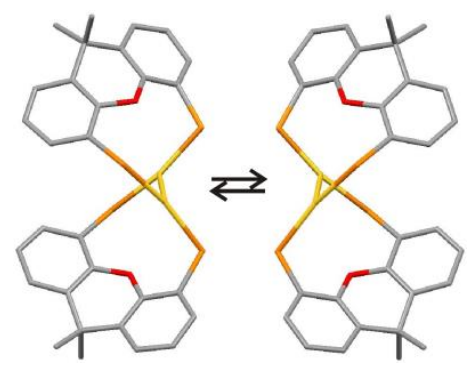

70 Fig. 1 Dynamic switching between figure-eight conformational enantiomers of double stranded $\left[\mathrm{Au}_{2} \mathrm{~L}_{2}\right]^{2+}$ helicate in solution. Colours: carbon, grey; oxygen, red; phosphorus, orange; gold, yellow; The phenyl groups are omitted for clarity.

Here we report several crystalline and amorphous forms of 75 this double stranded $\left[\mathrm{Au}_{2} \mathrm{~L}_{2}\right]^{2+}$ helicate, whose emission colours (blue, bluish green, yellow and red) from a single-component luminophore cover the whole visible spectral region. The existence of different crystalline forms of $\left[\mathrm{Au}_{2} \mathrm{~L}_{2}\right]^{2+}$ helicate provided a unique opportunity to study and understand the 80 relationship between crystal packing and multicolour luminescent properties. Furthermore, it also offers a notable example of an AIE active dinuclear double stranded helicate with solid-state photoemission that can be reversibly switched by external (mechanical and solvent vapour) stimuli.

85 To our knowledge, this metallosupramolecule is the first AIE active compound that exhibits both phase-dependent multicolour and reversible stimuli-responsive luminescent properties.

\section{Aggregation-Induced Emission}

Due to the conformational flexibility of the $\left[\mathrm{Au}_{2} \mathrm{~L}_{2}\right]^{2+}$ core, 90 compound $\mathbf{1}$ is practically non-luminescent in dichloromethane solution (Fig. 2a and b, at $0 \% \mathrm{Et}_{2} \mathrm{O}$ fraction). In order to check whether this double stranded $\left[\mathrm{Au}_{2} \mathrm{~L}_{2}\right]^{2+}$ helicate bearing aromatic rotors is AIE active, diethyl ether was added into dichloromethane solution of $\mathbf{1}$. a)

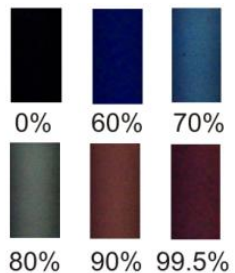

b)

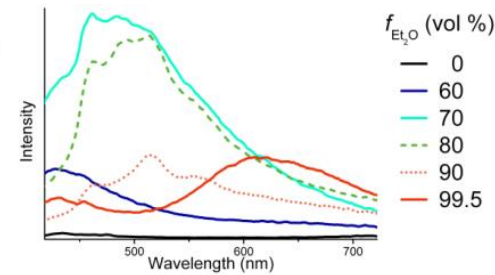


Fig. 2 a) Photographs taken under ultraviolet light (365 nm) and b) emission spectra of $\mathbf{1}$ in dichloromethane and its suspension in dichloromethane/diethyl ether mixtures $\left(\lambda_{\text {excit }}=365 \mathrm{~nm}\right)$.

Since 1 is insoluble in diethyl ether, increase in the diethyl ether 5 content causes the double stranded $\left[\mathrm{Au}_{2} \mathrm{~L}_{2}\right]^{2+}$ helicates to aggregate into ordered assemblies. Blue, bluish green, yellow and red emission colours (Fig. 2a) were featured in dichloromethane/diethyl ether mixtures containing various microstructures of $\mathbf{1}$ (Fig. S21 in ESI). Their emission spectra are 10 shown in Fig. 2b. When the diethyl ether fraction is increased to $60 \%$ the light-emission of 1 turns on, with an intensity boost of about 13-times and the photoluminescence spectrum indicates the formation of royal blue emitting $\left(\lambda_{\max }=430 \mathrm{~nm}\right)$ aggregates. Blue emitting ( $\lambda_{\max }=462$ and $495 \mathrm{~nm}$ ) assembly was present at $70 \%$ 15 diethyl ether fraction, and the emission intensity increases dramatically and reaches its maximum: it is about 55-fold higher than that in pure dichloromethane. At $80 \%$ diethyl ether content, blue and blue-green $\left(\lambda_{\max }=515 \mathrm{~nm}\right)$ emitting species were formed. Blue, blue-green and yellow $\left(\lambda_{\max }=560 \mathrm{~nm}\right)$ emitting 20 forms were formed in the mixture with $90 \%$ diethyl ether content. When the diethyl ether fraction is increased to $99.5 \%$, the emission spectrum indicates the presence of red $\left(\lambda_{\max }=616 \mathrm{~nm}\right)$ emitting aggregates, however, a small amount of royal blue emitting assemblies were also present. To our knowledge, this is 25 the first metallosupramolecule that exhibits five AIE colours (Fig. 2a) covering the whole visible spectral region.

The morphological properties of different forms of $\mathbf{1}$ obtained by varying the composition of the solvent mixtures were investigated by scanning electron microscopy (SEM) and 30 transmission electron microscopy (TEM). The TEM and SEM images (see the Supporting Information) indicate that different well-formed microstructures were formed in the mixtures with $60 \%-90 \%$ diethyl ether content, while spherical particles were present at $99.5 \%$ diethyl ether fraction. This simple method can 35 thus be used for the fabrication of micro-scale structures.

\section{Stimuli-Responsive Behaviour and Solid-State Luminescence}

To understand the relationship between the supramolecular structure of the double stranded $\left[\mathrm{Au}_{2} \mathrm{~L}_{2}\right]^{2+}$ helicate and its solid40 state emission properties, crystallization experiments were performed and photoluminescence spectra were recorded. As we already know, the vapour diffusion of diethyl ether into a concentrated dichloromethane solution of $\mathbf{1}$ at ambient temperature afforded large, brilliant, and beautifully formed ${ }_{45}$ crystals. ${ }^{46}$ However, in this previous work the crystallization conditions and the solid-state emission properties of the asobtained double stranded $\left[\mathrm{Au}_{2} \mathrm{~L}_{2}\right]^{2+}$ helicate were not examined. ${ }^{46}$

Now, crystallization of $\mathbf{1}$ from dichloromethane/diethyl ether mixtures was studied. Blue $\left(\mathbf{I}_{\mathbf{B}}\right)$, bluish green $\left(\mathbf{I I}_{\mathbf{G}}\right)$, yellow 50 emission $\left(\mathbf{I I I}_{\mathbf{Y}}\right)$ and red $\left(\mathbf{I V}_{\mathbf{R}}\right)$ emitting forms of double stranded $\left[\mathrm{Au}_{2} \mathrm{~L}_{2}\right]^{2+}$ helicate were obtained by variations in crystallization conditions (Fig. 3).

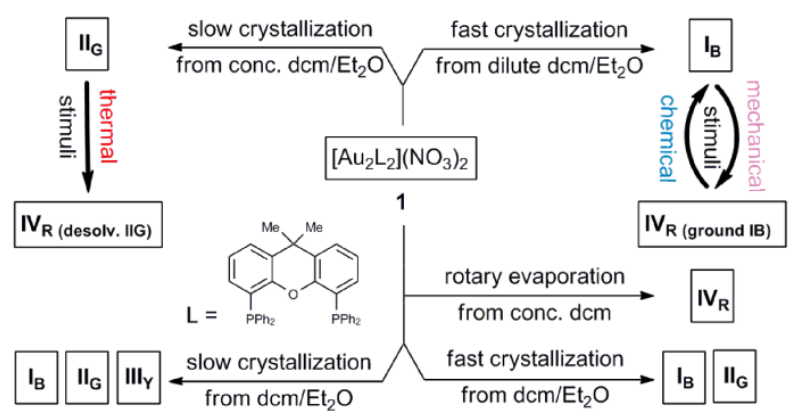

55 Fig. 3 A scheme illustrating the crystallization and phase transformation of blue $\left(\mathbf{I}_{\mathbf{B}}\right)$, bluish green $\left(\mathbf{I}_{\mathbf{G}}\right)$, yellow $\left(\mathbf{I I I}_{\mathbf{Y}}\right)$ and red $\left(\mathbf{I V}_{\mathbf{R}}\right)$ emitting forms of double stranded $\left[\mathrm{Au}_{2} \mathrm{~L}_{2}\right]^{2+}$ helicate.

Rapid crystallization from a dilute dichloromethane/diethyl ether solution of $\mathbf{1}\left(\mathrm{v} / \mathrm{v}\right.$ 1:2) afforded the blue-glowing $\mathbf{I}_{\mathbf{B}}$ form of ${ }_{60}$ double stranded $\left[\mathrm{Au}_{2} \mathrm{~L}_{2}\right]^{2+}$ helicate (Fig. 4a). Slow crystallization of a concentrated solution $\mathbf{1}(\mathrm{v} / \mathrm{v} 1: 1)$ produced large crystals $\mathbf{I I}_{\mathbf{G}}$ that exhibit bluish green emission (Fig. 4b). If a larger volume of diethyl ether was used (v/v 2:1), then crystals with bluish green $\mathbf{I I}_{\mathbf{G}}$ and blue $\mathbf{I}_{\mathbf{B}}$ emission were formed at room temperature (Fig. ${ }_{65} 4 \mathrm{c}$ ). The cooling of this dichloromethane/ diethyl ether mixture of $\mathbf{1}$ afforded crystals with blue $\mathbf{I}_{\mathbf{B}}$, bluish green $\mathbf{I I}_{\mathbf{G}}$ and yellow $\mathbf{I I I}_{\mathbf{Y}}$ emission (Fig. 4d). Thus, in this case blue, green and yellow emitting forms of double stranded $\left[\mathrm{Au}_{2} \mathrm{~L}_{2}\right]^{2+}$ helicate crystallize concomitantly. The concomitant crystallization of these different 70 solvates of $\mathbf{1}$ shows apparent similarity to concomitant crystallization of polymorphs. $^{51}$
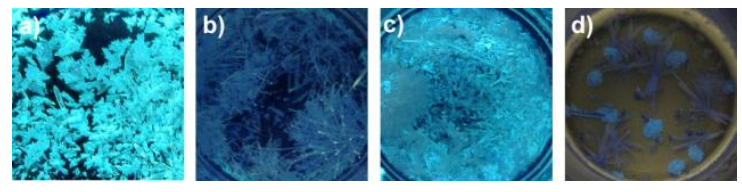

Fig. 4 Photographs taken under hand held UV lamp (365 nm) 75 illumination showing the crystallization of different forms of double stranded $\left[\mathrm{Au}_{2} \mathrm{~L}_{2}\right]^{2+}$ helicate from dichloromethane/diethyl ether mixtures: a) exclusive formation of blue emitting $\mathbf{I}_{B}$ crystals, b) exclusive formation of bluish green emitting $\mathbf{I I}_{\mathbf{G}}$ crystals, c) concomitant formation of $\mathbf{I}_{\mathbf{B}}$ and $\mathbf{I I}_{\mathbf{G}}$ crystals, d) concomitant formation of blue $\mathbf{I}_{\mathbf{B}}$, bluish green $\mathbf{I I}_{\mathbf{G}}$ and 80 yellow III $_{\mathbf{Y}}$ emitting crystals.

In addition to crystalline blue $\mathbf{I}_{\mathbf{B}}$ (Fig. 5a), bluish green $\mathbf{I I}_{\mathbf{G}}$ (Fig. 5b) and yellow $\mathbf{I I I}_{\mathbf{Y}}$ (Fig. 5c) emitting forms of $\left[\mathrm{Au}_{2} \mathrm{~L}_{2}\right]^{2+}$ helicate, a red emitting amorphous $\mathbf{I V}_{\mathbf{R}}$ form (Fig. 5d) was prepared by rotary evaporation of a dichloromethane solution of 851. 


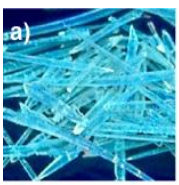

'B

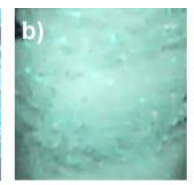

$\|_{G}$

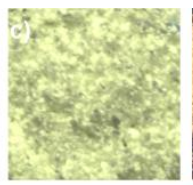

IIIY

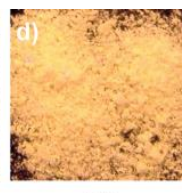

$\mathrm{IV}_{\mathrm{R}}$
Fig. 5 Photographs of blue $\mathbf{I}_{\mathbf{B}}$, bluish green $\mathbf{I I}_{G}$, yellow $\mathbf{I I I}_{\mathbf{Y}}$ emitting crystalline and red emitting $\mathbf{I V}_{\mathbf{R}}$ amorphous forms of double stranded $\left[\mathrm{Au}_{2} \mathrm{~L}_{2}\right]^{2+}$ helicate under $365 \mathrm{~nm}$ UV light illumination.

${ }_{5}$ Emission and excitation spectra of blue $\left(\mathbf{I}_{\mathbf{B}}\right)$, bluish green $\left(\mathbf{I}_{\mathbf{G}}\right)$, yellow emission (IIII) and red $\left(\mathbf{I V}_{\mathbf{R}}\right)$ emitting forms of double stranded $\left[\mathrm{Au}_{2} \mathrm{~L}_{2}\right]^{2+}$ helicate are shown in Fig. 6. The blue $\mathbf{I}_{\mathbf{B}}$ form shows an emission maximum at $490 \mathrm{~nm}$ (lifetime: $\tau_{1}=460 \mu \mathrm{s}$ $\left.(33 \%), \tau_{2}=1700 \mu \mathrm{s}(67 \%)\right)$. The $\mathbf{I I}_{\mathbf{G}}$ crystals exhibit bluish green 10 emission with $\lambda_{\max }$ at $520 \mathrm{~nm}$ and shorter emission lifetime $\left(\tau_{1}=\right.$ $\left.1.1 \mu \mathrm{s}(78 \%), \tau_{2}=15 \mu \mathrm{s}(22 \%)\right)$. The yellow-glowing III $_{\mathbf{Y}}$ crystalline form has $\lambda_{\max }$ at $570 \mathrm{~nm}(\tau=2.9 \mu \mathrm{s}(100 \%))$. The amorphous $\mathbf{I V}_{\mathbf{R}}$ form exhibits red emission with an emission maximum centred at $685 \mathrm{~nm}\left(\tau_{1}=3.6 \mu \mathrm{s}(34 \%), \tau_{2}=14 \mu \mathrm{s}\right.$ $15(66 \%))$. The blue emitting $\mathbf{I}_{\mathbf{B}}$ crystals retain their solvent of crystallization after standing in air for several months. Its desolvated $\mathbf{I}_{\mathbf{B} \text { (desolv.) }}$ form can be obtained by heating the $\mathbf{I}_{\mathbf{B}}$ crystals under reduced pressure at $90{ }^{\circ} \mathrm{C}$ for 20 minutes. This desolvated form of $\mathbf{I}_{\mathbf{B}}$ retains its original emission colour, and its 20 PXRD pattern (Fig. S2 and S3 in ESI) shows sharp lines suggesting retention of crystallinity upon solvent removal. The yellow III $_{\mathrm{Y}}$ crystals also preserve their original emission colour and crystallinity upon solvent removal (Fig. S6 and S7 in ESI).

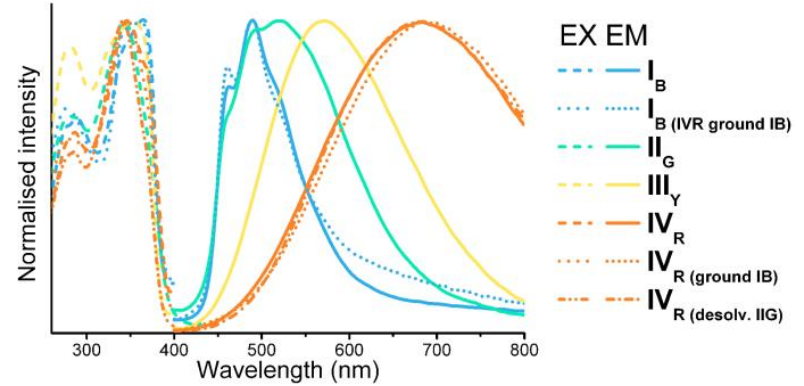

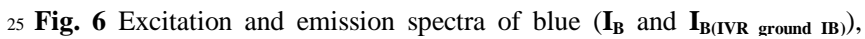
bluish green $\left(\mathbf{I I}_{\mathbf{G}}\right)$, yellow $\left(\mathbf{I I I}_{\mathbf{Y}}\right)$ and red $\left(\mathbf{I V}_{\mathbf{R}}, \mathbf{I V}_{\mathbf{R} \text { (ground } \mathbf{I B})}\right.$ and $\mathbf{I} \mathbf{V}_{\mathbf{R}(\text { desolv. }}$ IIG)) emitting forms of double stranded $\left[\mathrm{Au}_{2} \mathrm{~L}_{2}\right]^{2+}$ helicate.

In contrast to these extremely stable crystalline forms of double stranded $\left[\mathrm{Au}_{2} \mathrm{~L}_{2}\right]^{2+}$ helicate, the fast desolvation of the $\mathbf{I I}_{\mathrm{G}}$ 30 crystals changes their emission colour from bluish green through green and yellow to red (Fig. S4 in ESI). The as-resulting red emitting $\mathbf{I V}_{\mathbf{R}(\text { desolv IIG) }}$ form $\left(\lambda_{\max }=685 \mathrm{~nm}, \tau_{1}=3.9 \mu \mathrm{s}(43 \%), \tau_{2}\right.$ $=15 \mu \mathrm{s}(57 \%)$, Fig. 3) is amorphous (Fig. S5 in ESI), and its emission characteristics (Fig. 6) matched that of amorphous $\mathbf{I V}_{\mathbf{R}}$.

35 Apparently, this thermally induced crystalline-to-amorphous transformation exerts a dramatic influence on the photoluminescence properties. The loss of the original luminescence and the formation of a new emission colour upon desolvation has been rarely observed for gold(I) compounds. ${ }^{25}$

40 These different crystalline and amorphous forms obtained by slight modification of the crystallization conditions confirm the multi stimuli-responsive (solvent, concentration, temperature, etc.) behaviour of conformationally flexible double stranded $\left[\mathrm{Au}_{2} \mathrm{~L}_{2}\right]^{2+}$ helicate.
${ }_{45}$ The Effect of Molecular Conformations, Packing Arrangements and Interactions on the Solid-State Luminescence

The existence of different crystalline forms of this dinuclear double stranded $\left[\mathrm{Au}_{2} \mathrm{~L}_{2}\right]^{2+}$ helicate provided a unique opportunity 50 to study the effect of molecular conformations, packing arrangements and interactions on solid state luminescence properties.

Low-temperature X-ray diffraction of blue emitting $\mathbf{I}_{\mathbf{B}}(\mathbf{1} \cdot 2.64$ $\mathrm{CH}_{2} \mathrm{Cl}_{2}$ ) single crystal reveals that this new form of double 55 stranded $\left[\mathrm{Au}_{2} \mathrm{~L}_{2}\right]^{2+}$ helicate crystallized in a monoclinic $C 2 / c$ space group. In addition to low-temperature $(109 \mathrm{~K})$ measurements, X-ray diffraction was also performed at room temperature ( $\left.\mathbf{I}_{\mathbf{B}(\mathbf{R T})}, 295 \mathrm{~K}\right)$. These experiments show that the blue emitting crystals do not undergo temperature-driven phase 60 transition. The $\mathbf{I I}_{\mathbf{G}}\left(\mathbf{1} \cdot 4.69 \mathrm{CH}_{2} \mathrm{Cl}_{2}\right)$ needle crystal with bluish green emission has nearly the same crystal structure as the previously reported, ${ }^{46}$ however, with different solvent content. For the sake of comparison, its structural details are also given here. The single crystal X-ray diffraction of yellow emitting $\mathbf{I I I}_{\mathbf{Y}}$ 65 (1. $\left.1.90 \mathrm{CH}_{2} \mathrm{Cl}_{2} \cdot 0.59 \mathrm{H}_{2} \mathrm{O}\right)$ block crystal shows that this new form crystallized in a monoclinic $P 2_{1} / c$ space group. Crystal data and refinement parameters are summarized in Table S1 (ESI).

The low-temperature X-ray structural data (Table S2 in ESI) indicate that in these crystalline forms the dinuclear double 70 stranded $\left[\mathrm{Au}_{2} \mathrm{~L}_{2}\right]^{2+}$ helicate exists in a figure-eight conformation (Fig. 7), and the two ligand strands are folded with respect to the $\mathrm{Au} \cdots \mathrm{Au}$ axis, and held together by two $\mathrm{Au}(\mathrm{I})$ atoms at a distance of 2.838(1) $\AA\left(\mathbf{I}_{\mathbf{B}}\right), 2.837(1) \AA\left(\mathbf{I}_{\mathbf{B}(\mathbf{R T})}\right), 2.863(1) \AA\left(\mathbf{I I}_{\mathbf{G}}\right)$ and 2.841(1) $\AA\left(\right.$ III $\left._{\mathbf{Y}}\right)$, respectively. The luminescence from two75 coordinate gold(I) complexes, in many cases, have been associated with the presence of short $\mathrm{Au} \cdots \mathrm{Au}$ interactions. ${ }^{52}$

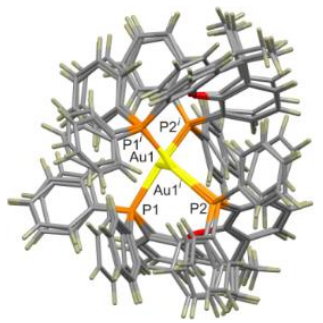

Fig. 7 Overlaid molecular structures of dinuclear double stranded $\left[\mathrm{Au}_{2} \mathrm{~L}_{2}\right]^{2+}$ helicate $\left(\mathbf{I}_{\mathrm{B}}, \mathbf{I I}_{\mathrm{G}}\right.$ and $\left.\mathbf{I I I} \mathbf{I}_{\mathrm{Y}}\right)$. Colours: carbon, grey; hydrogen, 80 dark-brown; oxygen, red; phosphorus, orange; gold, yellow.

At the molecular level, the major conformational difference is reflected in the $\mathrm{P}-\mathrm{Au}-\mathrm{P}$ angle, which is about $6.5^{\circ}$ smaller in $\mathbf{I I I}_{\mathbf{Y}}$ than in $\mathbf{I}_{\mathbf{B}}$, and about $5.2^{\circ}$ smaller in $\mathbf{I}_{\mathbf{G}}$ than in $\mathbf{I}_{\mathbf{B}}$. As a consequence, the dinuclear double stranded $\left[\mathrm{Au}_{2} \mathrm{~L}_{2}\right]^{2+}$ helicate in 85 both II $_{\mathbf{G}}$ and III $_{\mathbf{Y}}$ assume a compressed conformation with shorter intramolecular $\pi \cdots \pi$ interaction between the xanthene chromophores. As a result, the ligand backbone is more folded and tilted in both $\mathbf{I I}_{\mathbf{G}}\left(113.5(1)^{\circ}\right)$ and $\mathbf{I I I}_{\mathbf{Y}}\left(110.3(1)^{\circ}\right)$ than in $\mathbf{I}_{\mathbf{B}}$ $\left(103.2(1)^{\circ}\right)$ with respect to the $\mathrm{Au} \cdots \mathrm{Au}$ axis. Therefore, the ${ }_{90}$ molecules in both $\mathbf{I I}_{\mathbf{G}}$ and $\mathbf{I I I}_{\mathbf{Y}}$ assume a compressed conformation with shorter intramolecular $\pi \cdots \pi$ interaction between the xanthene chromophores (centroid-centroid distance = $5.08 \AA$ in $\mathbf{~ I I}_{\mathbf{G}}, 5.15 \AA$ in $\mathbf{I I I}_{\mathbf{Y}}$ and $5.52 \AA$ in $\mathbf{I}_{\mathbf{B}}$, for details see Figures S13, S17, S19 and Tables S3, S11, S14 in the Supporting 

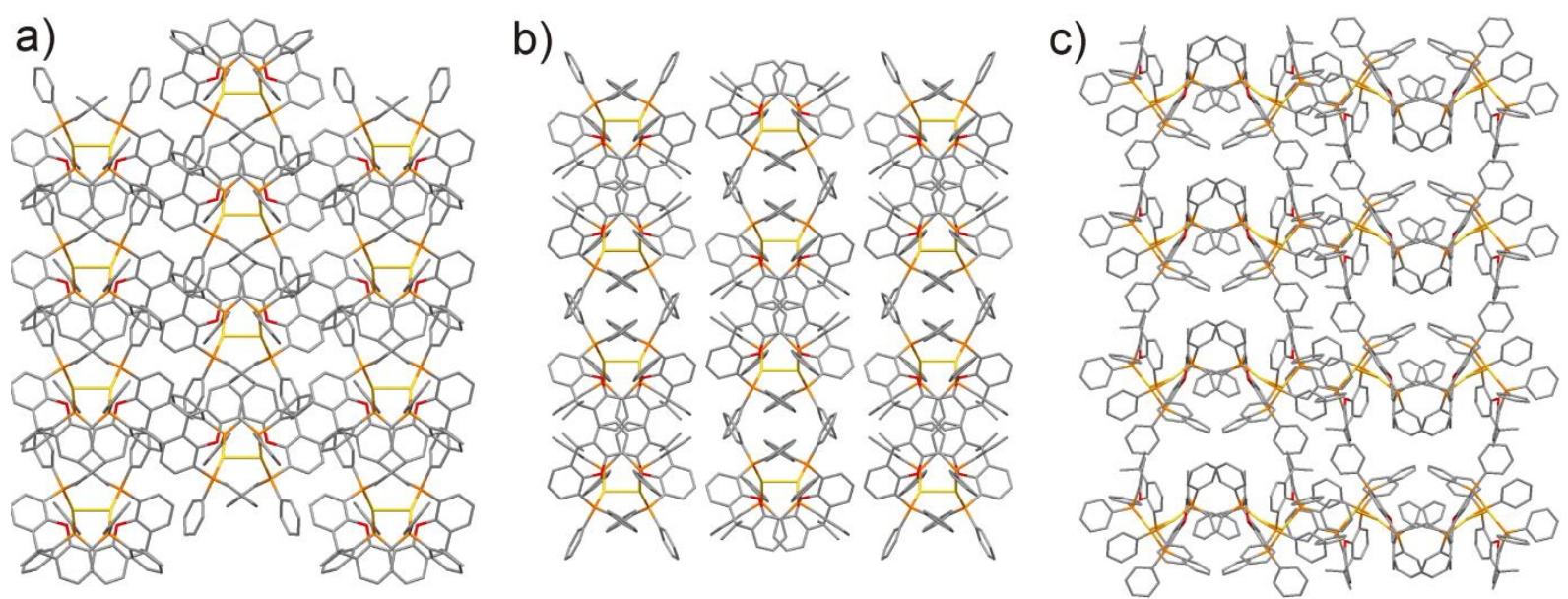

Fig. 8 A view of the crystal structures of a) $\mathbf{I}_{\mathbf{B}}$, b) $\mathbf{I I}_{\mathbf{G}}$ and c) $\mathbf{I I I}_{\mathbf{Y}}$ forms depicting the arrangement of dinuclear double stranded $\left[\mathrm{Au}_{2} \mathrm{~L}_{2}\right]^{2+}$ helicates. Colours: carbon, grey; oxygen, red; phosphorus, orange; gold, yellow.

5 Information). In solid-state some twist-shaped molecules may adopt a more twisted, less conjugated conformation making them to emit bluer light than in solution. ${ }^{12,13,17,18}$ We suppose that, at molecular level, thus, this lower degree of $\pi \cdots \pi$ conjugation between chromophores of dinuclear double stranded $\left[\mathrm{Au}_{2} \mathrm{~L}_{2}\right]^{2+}$ 10 helicate may be responsible for the blue-shifted emission of $\mathbf{I}_{\mathbf{B}}$ compared to those of $\mathbf{I I}_{\mathbf{G}}$ and $\mathbf{I I I}_{\mathbf{Y}}$ forms.

The different molecular packing in $\mathbf{I}_{\mathbf{B}}, \mathbf{I I}_{\mathbf{G}}$ and $\mathbf{I I I}_{\mathbf{Y}}$ crystalline forms of dinuclear double stranded $\left[\mathrm{Au}_{2} \mathrm{~L}_{2}\right]^{2+}$ helicate is shown in Fig. 8. In all these crystal structures, the bulkiness of the 15 diphosphine ligand prevents the formation of intermolecular aurophilic $\mathrm{Au} \cdots \mathrm{Au}$ interactions. Hence the molecular packing of these crystalline forms is primarily governed by $\pi \cdots \pi$ interactions between the dinuclear double stranded $\left[\mathrm{Au}_{2} \mathrm{~L}_{2}\right]^{2+}$ helicates (for details see the Figures S14, S18, S20 and Tables S4, S12, S15 in 20 the Supporting Information). Intermolecular $\pi \cdots \pi$ interactions, however, can have dramatic impact on the solid-state luminescence properties and can lead to red shifts in the emission spectra. ${ }^{7}$ In $\mathbf{I}_{\mathbf{B}}$ crystal, the $\left[\mathrm{Au}_{2} \mathrm{~L}_{2}\right]^{2+}$ helicates are packed into head-to-tail fashion to form columnar stacks (Fig. 8a) based on ${ }_{25} \pi \cdots \pi$ interactions between the xanthene chromophore and phenyl groups (centroid-centroid distance of 5.08 and $5.84 \AA$, for details see Figure S14 and Table S4 in the Supporting Information). These columnar stacks are also held together by $\pi \cdots \pi$ interactions (centroid-centroid distance of $3.70,4.84,4.87,5.32$ and $5.95 \AA$, 30 for details see Figure S14 and Table S4 in the Supporting Information). It should be noted that the molecular structure (see Figures S12, Table S2 in the Supporting Information), intra- and intermolecular $\pi \cdots \pi$ organization of $\left[\mathrm{Au}_{2} \mathrm{~L}_{2}\right]^{2+}$ helicates (see Figures S13-S16 and Tables S3, S4, S7, S8 in the Supporting 35 Information) are almost identical in low- (108 K, $\left.\mathbf{I}_{\mathbf{B}}\right)$ and hightemperature (295 K, $\mathbf{I}_{\mathbf{B}(\mathbf{R T})}$ ) forms. In order to gain a deeper insight into the effects of the conformational variations of the different molecular packing upon the intramolecular aurophilic interactions, we have performed quantum chemical DFT ${ }_{40}$ calculations for the geometries of crystalline structures $\mathbf{I}_{\mathbf{B}}, \mathbf{I I}_{\mathbf{G}}$ and $\mathbf{I I I}_{\mathbf{Y}}$, as well as for $\mathbf{I}_{\mathbf{B}(\mathbf{R T})}$. The calculated bond order index ${ }^{53}$ of the $\mathrm{Au} \cdots \mathrm{Au}$ show that the different environment in these structures has almost no effect in the case of $\mathbf{I}_{\mathbf{B}}, \mathbf{I}_{\mathbf{G}}(\mathrm{BO}=0.42$,
0.43), while slightly strengthens the aurophilic interaction in 45 structure III $_{\mathbf{Y}}(\mathrm{BO}=0.48)$ (for details see the Supporting Information). In bluish green emitting $\mathbf{I I}_{\mathbf{G}}$ crystals, the dinuclear $\left[\mathrm{Au}_{2} \mathrm{~L}_{2}\right]^{2+}$ helicates are arranged into dimers through slipped $\pi \cdots \pi$ interactions between the xanthene chromophores (centroidcentroid distance of $5.36 \AA$, and slippage of $1.64 \AA$ ) and xanthene 50 and phenyl rings (centroid-centroid distance of $5.80 \AA$ ), showing a brick-wall packing pattern (Fig. 8b). Here each dimer overlaps via additional $\pi \cdots \pi$ interactions with two halves of dimers in the row above and below (centroid-centroid distance of 4.41, 4.93, 4.98, 5.47 and $5.99 \AA$, for details see Figure S18 and Table S12 in 55 the Supporting Information). In fact, the dinuclear double stranded $\left[\mathrm{Au}_{2} \mathrm{~L}_{2}\right]^{2+}$ helicates are more slipped in $\mathbf{I I}_{\mathbf{G}}$ than in $\mathbf{I}_{\mathbf{B}}$. As a result of this somewhat increased $\pi$-conjugation, the emission maximum of $\mathbf{I I}_{\mathbf{G}}$ displays a red shift compared with that of $\mathbf{I}_{\mathbf{B}}$. However, in $\mathbf{I}_{\mathbf{B}}$ crystal there are additional $\mathrm{C}-\mathrm{H} \cdots \pi$ interactions 60 (Table S5 in the Supporting Information). In yellow emitting $\mathbf{I I I}_{\mathbf{Y}}$ crystal, the twisted-core $\mathrm{Au}_{2} \mathrm{~L}_{2}$ units form helical chains (Fig. 8c) based on $\pi \cdots \pi$ interactions between the xanthene chromophores and phenyl groups (centroid-centroid distance of 3.90, 4.79, 5.26, 5.48, 5.60, 5.61, 5.67, 5.75 and $5.78 \AA$, for details see ${ }_{65}$ Figure S20 and Table S15 in the Supporting Information). These helices are also held together by $\pi \cdots \pi$ interactions (centroidcentroid distance of $4.85,5.25,5.41,5.53,5.64$ and $5.83 \AA$, for details see Figure S20 and Table S15 in the Supporting Information). The higher degree of $\pi \cdots \pi$ conjugation between 70 dinuclear $\left[\mathrm{Au}_{2} \mathrm{~L}_{2}\right]^{2+}$ helicates together with a large set of additional multiple aromatic interactions $(\mathrm{C}-\mathrm{H} \cdots \pi, \mathrm{C}-\mathrm{H} \cdots \mathrm{O}$ and $\mathrm{N}-\mathrm{O} \cdots \pi$, Tables S16-S18 in the Supporting Information) may induce the more red-shifted emission of III $_{\mathbf{Y}}$.

The emission of red emitting amorphous $\mathbf{I V}_{\mathbf{R}}$ is 75 bathochromically shifted compared to all three crystalline forms of dinuclear double stranded $\left[\mathrm{Au}_{2} \mathrm{~L}_{2}\right]^{2+}$ helicate. In contrast to crystal structures, where the relative orientations of molecules are strictly defined, a large distribution of molecular packing is possible in amorphous solids. ${ }^{17}$ Within this random packing 80 arrangements, a larger set of favourable $\pi \cdots \pi$ interactions between the chromophores and phenyl rotors of $\left[\mathrm{Au}_{2} \mathrm{~L}_{2}\right]^{2+}$ helicates together with other noncovalent interactions may be 
responsible for the red emission observed for the amorphous $\mathbf{I} \mathbf{V}_{\mathbf{R}}$.

Similarity among the emission spectrum of the blue emitting $\mathbf{I}_{\mathbf{B}}$ crystal at room temperature and glassy ethanol solution of $\mathbf{1},{ }^{46}$ as well as, diphosphine ligand at $77 \mathrm{~K}^{46}$ suggests that in this case, 5 the luminescence occurred mainly from single molecules. Moreover, on the basis of the long lifetime of $\mathbf{I}_{\mathbf{B}}$ this blue emission can be attributed to the phosphorescence from the intraligand type excited state. On the other hand, the shorter lifetimes and red-shifted emissions of crystalline $\mathbf{I I}_{\mathbf{G}}$ and $\mathbf{I I I}_{\mathbf{Y}}$ as 10 well as amorphous $\mathbf{I} \mathbf{V}_{\mathbf{R}}$ compared with that of crystalline $\mathbf{I}_{\mathbf{B}}$ form indicate that the increasing number of intra- and mainly intermolecular $\pi \cdots \pi$ interactions lock and rigidify the figure-eight conformation of the double stranded $\left[\mathrm{Au}_{2} \mathrm{~L}_{2}\right]^{2+}$ helicate and opens up new radiative pathways. Thus, in accordance with AIE 15 phenomenon, the conformational motions and rotation of aromatic rings are restricted in solid-state, rendering the $\left[\mathrm{Au}_{2} \mathrm{~L}_{2}\right]^{2+}$ helicate emissive.

The crystallographic data are not only in full agreement with spectroscopic characteristic but make it possible to rationalize the 20 photoluminescent properties of these double stranded $\left[\mathrm{Au}_{2} \mathrm{~L}_{2}\right]^{2+}$ helicates. Thus, based on the single crystal X-ray analysis now is obvious that different molecular conformations, packing arrangements result in different non-covalent interactions between dinuclear double stranded $\left[\mathrm{Au}_{2} \mathrm{~L}_{2}\right]^{2+}$ helicates in blue, 25 bluish green and yellow emitting crystals. These factors have played the most significant role in tailoring the solid-state luminescent properties of these dinuclear $\left[\mathrm{Au}_{2} \mathrm{~L}_{2}\right]^{2+}$ helicates. In other words, different emission colours based on a same emitting $\left[\mathrm{Au}_{2} \mathrm{~L}_{2}\right]^{2+}$ species were achieved owing to the conformational 30 flexibility of the double stranded helicate, which in its turn, generates diverse molecular conformations and packing arrangements modulated mainly by $\pi \cdots \pi$ interactions in the solidstate.

\section{Stimuli-Responsive Luminescence}

35 As we already mentioned, up to now, only two gold(I) complexes that exhibit reversible mechanochromic luminescence have been reported. ${ }^{42,43}$ A molecular-level understanding of these mechanically induced reversible luminescence changes in these gold complexes remains unclear. ${ }^{2}$ It was, however, suggested 40 that the grinding-induced new emission might arises from emerging aurophilic $\mathrm{Au} \cdots \mathrm{Au}$ interactions or distortion of the molecular conformation. ${ }^{2,42,43}$ Very recently, it was reported that a small mechanical stimulus can trigger a single-crystal-to-singlecrystal transformation of a $\left(\mathrm{C}_{6} \mathrm{H}_{5}\right) \mathrm{Au}\left(\mathrm{CNC}_{6} \mathrm{H}_{5}\right)$ complex. ${ }^{44}$ The 45 transformation proceeds through self-replication and blue photoluminescence of the crystal changes into yellow one after mechanical stimulus. ${ }^{44}$ Our double stranded $\left[\mathrm{Au}_{2} \mathrm{~L}_{2}\right]^{2+}$ helicate having different crystalline and amorphous forms seems an ideal candidate to study the origin of mechanochromic luminescent 50 colour switching.

The molecular packing-sensitive solid-state emission of dinuclear double stranded $\left[\mathrm{Au}_{2} \mathrm{~L}_{2}\right]^{2+}$ helicate was evident upon mechanical perturbation. When the blue emitting $\mathbf{I}_{\mathbf{B}}$ crystals were ground the luminescence colour of the as-formed powder ${ }_{55} \mathbf{I} \mathbf{V}_{\mathbf{R} \text { (ground IB) }}$ changed to red (Fig. 9a). Its emission spectrum $\left(\lambda_{\max }\right.$ $\left.=690 \mathrm{~nm}, \tau_{1}=3.4 \mu \mathrm{s}(33 \%), \tau_{2}=14 \mu \mathrm{s}(67 \%)\right)$ is identical to that of amorphous $\mathbf{I V}_{\mathbf{R}}$ (Fig. 6). The red shift of $200 \mathrm{~nm}$ in luminescence emission upon grinding is unprecedented among mechanochromic luminescent compounds. ${ }^{9,15}$
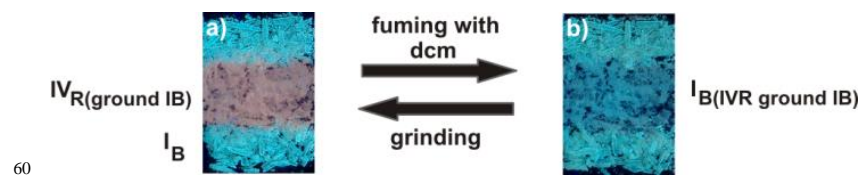

Fig. 9 Reversible stimuli-responsive luminescence of $\mathbf{I}_{\mathbf{B}}$ : a) red emitting $\mathbf{I V}_{\mathbf{R}}$ (ground IB) obtained by grinding the blue luminescent $\mathbf{I}_{\mathbf{B}}$ and $\mathbf{b}$ ) reversion to blue luminescent $\mathbf{I}_{\mathbf{B}(\mathbf{I V R} \text { ground IB) }}$ after fuming $\mathbf{I V}_{\mathbf{R} \text { (ground IB) }}$ with 65 dichloromethane

The peak intensities significantly decreased and broadened in the PXRD pattern of this $\mathbf{I} \mathbf{V}_{\mathbf{R}(\text { ground IB) }}$ powder, however, the presence of some peaks indicates an amorphous phase with only shortrange order (Fig. 10b). The luminescence lifetimes of the 70 crystalline $\mathbf{I}_{\mathbf{B}}$ and amorphous $\mathbf{I V}_{\mathbf{R}(\text { ground IB) }}$ forms indicate that the non-radiative decay is more suppressed in crystalline state, resulting in significantly longer lifetime than in amorphous state.

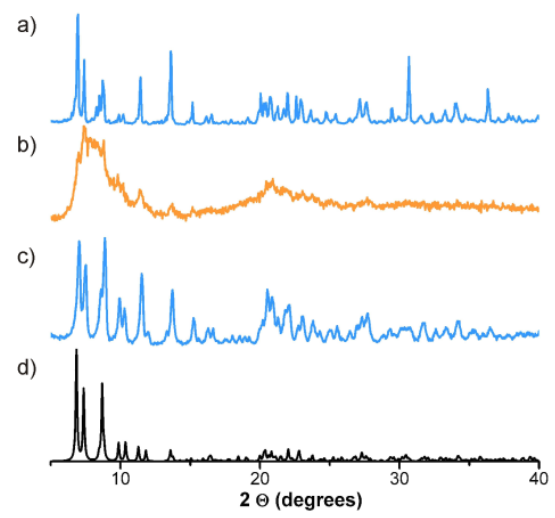

Fig. 10 Powder X-ray diffraction patterns of a) the as-synthesized 75 crystalline $\mathbf{I}_{\mathbf{B}}$, b) amorphous $\mathbf{I V}_{\mathbf{R}(\text { ground }}$ IB) obtained by grinding the crystalline $\mathbf{I}_{\mathbf{B}}$, c) crystalline $\mathbf{I}_{\mathbf{B}(\mathbf{I V R} \text { ground } \mathbf{I B})}$ obtained by exposing to dichloromethane vapour the amorphous $\mathbf{I} \mathbf{V}_{\mathbf{R}(\text { ground IB })}$ and the d) simulated diffraction pattern of $\mathbf{I}_{\mathbf{B}}$ based on the single crystal structure.

The original blue luminescence restored when the red emitting

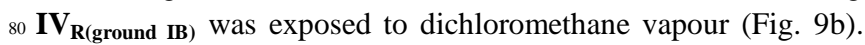

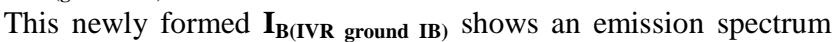
identical to that of $\mathbf{I}_{\mathbf{B}}$ (Fig. 6) and sharp diffraction peaks emerge again (Fig. 10c). Thus, the exposure to solvent vapours induces the crystallization of amorphous powder, and this amorphous-to85 crystalline transformation is accompanied by significant luminescence shift. The dynamic switching process between blue and red luminescence can be repeated many times by using subsequent grinding and solvent fuming cycles (Fig. 11).

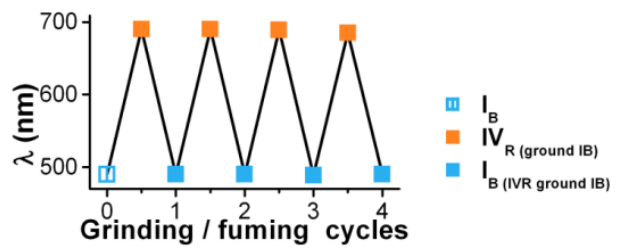

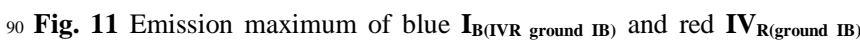
emitting forms measured in four consecutive grinding/fuming cycles showing the reversibility of the mechanochromic luminescence.

We next conducted the mechanical triggering experiment with 
a much considerable/higher mechanical stimulus involving ballmilling. We implemented the ball-milling technique to further study these crystalline-to-amorphous (CTA) and amorphous-tocrystalline (ATC) transformations. The crystalline $\mathbf{I}_{\mathbf{B}}$ complex 5 was ball-milled and the transformation was monitored by PL spectroscopy. Remarkably, as shown by PL spectra (Figure 12), the crystalline $\mathbf{I}_{\mathrm{B}}$ complex was amorphized by ball-milling after 9 minutes, and the resulting $\mathbf{I} \mathbf{V}_{\mathbf{R}(\text { ball-milled IB) }}$ powder, was identical to red emitting amorphous $\mathbf{I V}_{\mathbf{R}}$ form.

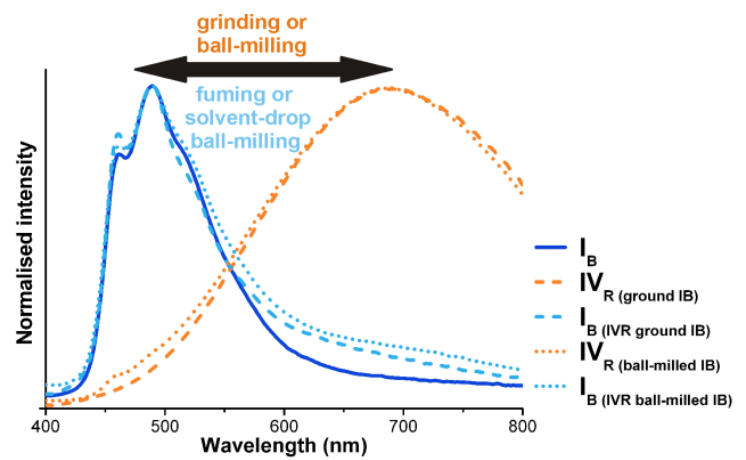

10

Fig. 12 The emission spectra of blue-emitting $\mathbf{I}_{\mathbf{B}}, \mathbf{I}_{\mathbf{B}(\mathbf{I V R} \text { ground IB) }}$ and $\mathbf{I}_{\mathbf{B}(\mathrm{IVR}}$ ball-milled IB), as well as, red-emitting $\mathbf{I V}_{\mathbf{R} \text { (ground }}$ IB) and $\mathbf{I V}_{\mathbf{R} \text { (ball-milled IB) }}$ samples.

15 Further we probed the solvent-drop assisted ball-milling to achieve amorphous to crystalline transformations. The PL spectra (Fig. 12) showed that the amorphous $\mathbf{I V}_{\mathbf{R}(\text { ball-milled IB) }}$ sample obtained by mechanochemical CTA transformation was easily transformed/converted back into crystalline blue emitting $\mathbf{I}_{\mathbf{B}(\mathbf{I V R}}$ 20 ball-milled IB) form by grinding with dichloromethane for 2 minutes. To our knowledge, this is the first precedent, when the reversion to original luminescence colour has been achieved by solventdrop assisted ball-milling process. These CTA and ATC phase changes were accompanied by a dramatic luminescence colour 25 change. These results indicate that the mechanochemical wet or solvent-drop assisted ball-milling techniques can be used to perform reversible CTA and ATC transformations.

To show more clearly the mechanochromic and vapochromic luminescent properties of our dinuclear double stranded $\left[\mathrm{Au}_{2} \mathrm{~L}_{2}\right]^{2+}$ 30 helicate, $\mathbf{I}_{\mathbf{B}}$ in $\mathrm{n}$-hexane suspension was deposited on a filter paper, which further was dried at ambient temperature. As shown in Fig. 13, various kinds of symbols can be recorded by handwriting on this paper using a spatula. These patterns can be erased by solvent fuming and new marks can be recorded.
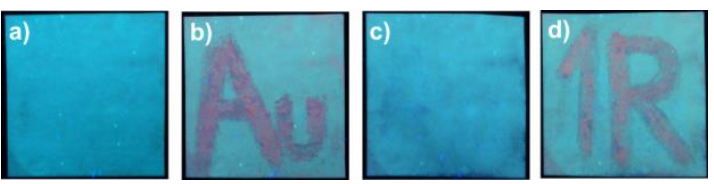

Fig. 13 Luminescent images obtained under $365 \mathrm{~nm}$ UV irradiation of a) a suspension of $\mathbf{I}_{\mathbf{B}}$ deposited on a filter paper, b) chemical symbol „Au” was written on the paper, c) erasing the pattern by fuming with 40 dichloromethane vapours, d) recording ,1R" on the same position.

This simple experiment reveals a reversible recording-erasing process, which can be repeated by alternating amorphous-to- crystalline transformations. These intriguing dynamic phase change properties render this material particularly attractive for a 45 wide range of applications such as optical recording, security inks and papers, latent image developers as well as pressure, deformation and volatile organic compound sensors.

Based on PXRD results (Fig. 10), now is evident that the stimuli-responsive luminescence of this conformationally flexible 50 double stranded $\left[\mathrm{Au}_{2} \mathrm{~L}_{2}\right]^{2+}$ helicate proceeds through a mechanism that allows dynamic switching between crystalline and amorphous states. Thus, owing to the twisted-molecular shape of the dinuclear $\left[\mathrm{Au}_{2} \mathrm{~L}_{2}\right]^{2+}$ helicate, the molecular packing is loose and under continuous mechanical stress the entire crystal 55 can be easily destroyed leading to the formation of an amorphous phase. In the crystalline state, the molecules are closely packed, however, they can escape the mechanical stimulus by farreaching migrations. ${ }^{54}$ In accordance with PXRD spectrum, however, some memory of blue luminescent crystalline form 60 appears to be retained in amorphous form obtained by grinding. This memory of molecular self-assembly patterns would direct the conformationally mobile dinuclear $\left[\mathrm{Au}_{2} \mathrm{~L}_{2}\right]^{2+}$ helicate towards the reformation of crystalline order. Thus, in the presence of solvent vapours, the $\left[\mathrm{Au}_{2} \mathrm{~L}_{2}\right]^{2+}$ helicates have increased molecular 65 mobility to allow the conformational changes necessary for the reformation of crystalline order. Molecular mobility is generally thought to be a key factor in determining the crystallization tendency from the amorphous state. ${ }^{55,56}$ In fact, however, this stimuli-responsive dinuclear double stranded $\left[\mathrm{Au}_{2} \mathrm{~L}_{2}\right]^{2+}$ helicate is 70 a phase change material $^{57}$ that can be switched reversibly between crystalline and amorphous phases having distinctly different luminescent properties.

It becomes clear that the stimuli-responsive luminescence of conformationally flexible double stranded $\left[\mathrm{Au}_{2} \mathrm{~L}_{2}\right]^{2+}$ helicate is 75 associated with reversible CTA and ATC transformations. Surprisingly, in accordance with the reported PXRD data, ${ }^{42,43}$ the previously reported gold(I) complexes exhibiting reversible mechanochromic luminescent properties are in fact phase change materials. On the basis of these results, it seems plausible that the 80 mechanochromic luminescence of gold-containing compounds proceeds through a mechanism that allows dynamic switching between crystalline and amorphous states. The ATC transformations are significant both from conceptual as well as application perspectives. ${ }^{57,58}$ They are familiar in phase-change 85 materials, pharmaceutical compounds and biomineralization. ${ }^{57,58}$

We further tested the applicability of our findings on a previously synthesized double-stranded $\left[\mathrm{Au}_{2} \mathrm{~L}_{2}\right]^{2+}$ helicate consisting of gold(I) atom and 4,6-bis(diphenylphosphino)phenoxazine ligand (L'). ${ }^{48}$ The luminescence colour of this 90 dinuclear $\left[\mathrm{Au}_{2} \mathrm{~L}_{2}\right]^{2+}$ helicate can also be switched reversibly from ochre to dark red through repeated ball-milling and methanol-assisted ball-milling cycles (Fig. 14).
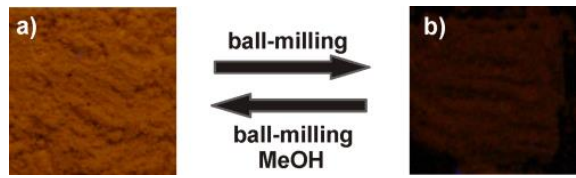

95 Fig. 14 Reversible luminescence colour switching of dinuclear $\left.\left[\mathrm{Au}_{2} \text { (nixantphos) }\right)_{2}\right]^{2+}$ helicate from ochre to dark red through repeated mechanical and chemical (solvent) stimuli. 
These findings could provide a novel concept for the design of AIE active stimuli-responsive solid-state luminescent materials that is based on luminescent gold(I) atoms and diphosphine ligands bearing aromatic rotors. The success of this approach 5 relies on the variety of diphosphine ligands which could afford a range of conformationally flexible double-stranded $\left[\mathrm{Au}_{2} \mathrm{~L}_{2}\right]^{2+}$ helicates whose emission colour can be tuned or switched over the whole visible spectral region.

\section{CONCLUSIONS}

10 In summary, we showed that a conformationally flexible dinuclear double-stranded $\left[\mathrm{Au}_{2} \mathrm{~L}_{2}\right]^{2+}$ helicate constructed from luminescent $\operatorname{gold}(\mathrm{I})$ atoms and diphosphine ligands bearing aromatic rotors could exhibit unique stimuli responsive luminescent properties. Different crystalline and amorphous 15 forms of dimeric $\left[\mathrm{Au}_{2} \mathrm{~L}_{2}\right]^{2+}$ helicate whose emission colours (blue, bluish green, yellow and red) from a single-component luminophore cover the whole visible spectral region were obtained by slight modification of the crystallization condition.

Based on the single crystal X-ray analysis is obvious that 20 different molecular conformations, packing arrangements result in different non-covalent interactions between dinuclear double stranded $\left[\mathrm{Au}_{2} \mathrm{~L}_{2}\right]^{2+}$ helicates in blue, bluish green and yellow emitting crystals. These factors played the most significant role in tailoring the solid-state luminescent properties. This 25 metallosupramolecule offers a notable example of an AIE active compound with solid-state photoemission that can be reversibly switched by external (mechanical and chemical) stimuli. These results may provide the basis for improved and innovative design strategies for novel stimuli-responsive luminescent and phase 30 change materials.

\section{EXPERIMENTAL SECTION}

Materials. All chemicals and solvents used for the syntheses were of reagent grade. The solvents for synthesis were used without further purification. All manipulations were performed in 35 air. 1 was prepared according to a published procedure. ${ }^{46}$

Production of blue $\mathbf{I}_{\mathbf{B}}$ luminescent crystals: A $83.8 \mathrm{mg}(0.05$ $\mathrm{mmol}$ ) sample of $\mathbf{1}$ was dissolved in $10 \mathrm{~mL}$ of dichloromethane. The as-resulting clear solution was then filtered through Celite. 40 To this filtrate $20 \mathrm{~mL}$ of diethyl ether was carefully layered. Blue luminescent $\mathbf{I}_{\mathbf{B}}$ crystals were obtained overnight. The product was filtered off and washed with cold diethyl ether. Elemental analysis calcd (\%) for $\mathbf{I}_{\mathbf{B}}\left(\mathbf{1} \cdot 2.64 \mathrm{CH}_{2} \mathrm{Cl}_{2}\right): \mathrm{C} 50.99, \mathrm{H} 3.68, \mathrm{~N}$ 1.47; found: C 50.71, H 3.45, N 1.52. IR data $\left(\mathrm{cm}^{-1}\right): 3056(\mathrm{~m}$, 45 br), 2971 (m, br), 1479 (m), 1435 (m), 1403 (s), 1343 (s, br), 1221 (s), 1151 (m), 1095 (s), 998 (m), 875 (m), 829 (m), 790 (m), 731 (m, br), 688 (s).

Production of bluish green $\mathbf{I I}_{\mathbf{G}}$ luminescent crystals: A 427.2 $50 \mathrm{mg}(0.255 \mathrm{mmol})$ of $\mathbf{1}$ was dissolved in $15 \mathrm{~mL}$ of dichloromethane. The as-resulting clear solution was then filtered through Celite. To this filtrate $15 \mathrm{~mL}$ of diethyl ether was carefully layered and the mixture was placed in a refrigerator (4 $\left.{ }^{\circ} \mathrm{C}\right)$. Bluish green luminescent $\mathbf{I I}_{\mathbf{G}}$ crystals were obtained 55 overnight. However, small amounts of blue luminescent crystals were also formed alongside these crystals. The product was filtered off and washed with cold diethyl ether. Elemental analysis found $(\%)$ for $\mathbf{I I}_{\mathbf{G}}$ : $\mathbf{C} 53.91, \mathrm{H} 3.62, \mathrm{~N} 1.68$. This suggests that $\mathbf{I I}_{\mathbf{G}}$ contains 1.2 moles of dichloromethane 60 (Elemental analysis calcd (\%) for $\mathbf{1} \cdot 1.2 \mathrm{CH}_{2} \mathrm{Cl}_{2}: \mathrm{C} 53.53, \mathrm{H} 3.77$, $\mathrm{N}$ 1.58). This value is lower than the crystallographically predicted dichloromethane content. This is due to the rapid solvent loss that occurs during standing.

${ }_{65}$ Production of the mixture of blue $\mathbf{I}_{\mathrm{B}}$, bluish green $\mathbf{I I}_{\mathrm{G}}$ and yellow III $_{Y}$ luminescent crystals: A $427.2 \mathrm{mg}(0.255 \mathrm{mmol})$ of $\mathbf{1}$ was dissolved in $15 \mathrm{~mL}$ of dichloromethane. The as-resulting clear solution was then filtered through Celite. To this filtrate 30 $\mathrm{mL}$ of diethyl ether was carefully layered and the mixture was 70 placed in a refrigerator $\left(4{ }^{\circ} \mathrm{C}\right)$. Blue $\mathbf{I}_{\mathbf{B}}$, bluish green $\mathbf{I I}_{\mathbf{G}}$ and yellow III $_{\mathbf{Y}}$ luminescent crystals were obtained overnight. The crystals were filtered off and washed with cold diethyl ether. The different crystalline forms were separated manually under $365 \mathrm{~nm}$ UV lamp. Elemental analysis calcd (\%) for $\mathbf{I I I}_{\mathbf{Y}}\left(\mathbf{1} \cdot 1.9 \mathrm{CH}_{2} \mathrm{Cl}_{2}\right)$ : ${ }_{75} \mathrm{C} 52.25, \mathrm{H} 3.72, \mathrm{~N} 1.53$; found: C 52.71, H 3.42, N 1.71. IR data $\left(\mathrm{cm}^{-1}\right)$ : 3445 (m, br), 3053 (m, br), $1408(\mathrm{~m}), 1435$ (s), 1402 (s), 1325 (m), 1306 (m), 1209 (s), 1099 (s), 745 (s), 711 (m), 688 (s).

Production of the mixture of blue $I_{B}$ and bluish green $\mathbf{I I}_{G}$ 80 luminescent crystals: A $427.2 \mathrm{mg}(0.255 \mathrm{mmol})$ of $\mathbf{1}$ was dissolved in $15 \mathrm{~mL}$ of dichloromethane. The as-resulting clear solution was then filtered through Celite. To this filtrate $30 \mathrm{~mL}$ of diethyl ether was carefully layered. Blue $\mathbf{I}_{\mathbf{B}}$ and bluish green $\mathbf{I I}_{\mathbf{G}}$ luminescent crystals were obtained overnight. The different 85 crystalline forms were separated manually under $365 \mathrm{~nm} \mathrm{UV}$ lamp.

Production of red luminescent amorphous $\mathbf{I V}_{\mathbf{R}}$ form: A 410 $\mathrm{mg}(0.245 \mathrm{mmol})$ of $\mathbf{1}$ was dissolved in $8 \mathrm{~mL}$ of dichloromethane. ${ }_{90}$ The as-resulting clear solution was then filtered through Celite. The red emitting amorphous $\mathbf{I V}_{\mathbf{R}}$ form was prepared by rotary evaporation of this dichloromethane solution. Elemental analysis calcd (\%) for $\mathbf{I V}_{\mathbf{R}}$ : C 55.92, H 3.85, N 1.67; found: C 55.72, H 3.87, N 1.70. IR data $\left(\mathrm{cm}^{-1}\right)$ : 3434 (m, br), 3057 (m, br), $2977(\mathrm{~m}$, 95 br), 1480 (m), 1436 (m), 1402 (s), 1332 (m, br), 1216 (m), 1096 (m), 998 (m), 741 (m), 687 (m).

Ball-milling experiment: CTA transformation was performed using $100 \mathrm{mg}$ of crystalline sample placed into $5 \mathrm{~mL}$ agate jar 100 with two $7 \mathrm{~mm}$ diameter agate balls was ground with a Retsch MM400 shaker mill operating at $25 \mathrm{~Hz}$ for the specified time. The ATC transformation was performed on the amorphous sample obtained from CTA transformation with the addition of $30 \mu \mathrm{L}$ dichloromethane. After every 1 minute of milling, a small 105 amount of sample was taken out of the jar, and its PL spectrum was recorded.

\section{Physical Measurements}

The elemental analysis has been carried out with an Elementar Vario EL III apparatus. Infrared spectra were recorded in the 550 110 to $4000 \mathrm{~cm}^{-1}$ spectral range on a Varian 2000 FT-IR spectrometer equipped with Golden Gate Single Reflection Diamond ATR (Attenuated Total Reflection) accessory (Specac). Steady state 
and time-resolved luminescence measurements were carried out on an Edinburgh Instrument FLSP920 spectrofluorimeter. Spectral corrections were applied using excitation and emission correction functions of the instrument. The solid-state room 5 temperature emission studies were conducted on finely ground powder samples placed on a Quartz Suprasil plate in a front face sample holder. Photoluminescence spectra of $\mathbf{I I}_{\mathbf{G}}$ were recorded on crystals placed with a small amount of mother liquor in a 5 $\mathrm{mm}$ quartz tube. Longpass filters were used to exclude the 10 scattered excitation light. The excitation light source was a $\mu \mathrm{F} 900 \mathrm{H}$ xenon flashlamp (pulse duration: $2 \mu$ at FWHM) for the luminescent lifetime measurements. Powder diffractograms were produced with $\mathrm{Cu}-K_{\alpha}$ radiation on a vertical high-angle Philips PW 1050 powder diffractometer.

\section{${ }_{15}$ X-ray Crystallography and Data Collection}

Crystals were mounted in Paratone- $\mathrm{N}$ oil within a conventional cryo-loop, and intensity data were collected on a Rigaku R-AXIS RAPID image plate diffractometer $\left(\lambda\left(\right.\right.$ Mo- $K_{\alpha}$ radiation $)=$ $0.71070 \AA$ ), fitted with an X-stream low temperature attachment.

20 Several scans in the $\varphi$ and $\omega$ direction were made to increase the number of redundant reflections, which were averaged over the refinement cycles. The structures were solved by direct method $(S I R 92)^{59}$ and refined by full-matrix least-squares (SHELXL97) ${ }^{60}$ All calculations were carried out using the Win $G X$ package 25 of crystallographic programs. ${ }^{61}$ In complex $\mathbf{I}_{\mathbf{B}}$, we modelled a total of 2.64 dichloromethane molecules, one of them being disordered over two positions. A total of 2.26 dichloromethane molecules were modelled in complex $\mathbf{I}_{\mathbf{B}(\mathbf{R T})}$, one of them being disordered over two positions. In complex $\mathbf{I I}_{\mathbf{G}}$, a total of 4.69 30 dichloromethane molecules were modelled. A total of 1.90 dichloromethane molecules and an uncoordinated water molecule with site occupancy of 0.59 were found in the crystal structure of III $_{\mathbf{Y}}$. The methyl $\mathrm{H}$ atoms were constrained to an ideal geometry $(\mathrm{C}-\mathrm{H}=0.96 \AA)$ with $\mathrm{U}_{\text {iso }}(\mathrm{H})=1.5$ (methyl), but were allowed to 35 rotate freely about the $\mathrm{C}-\mathrm{C}$ bonds. All remaining $\mathrm{H}$ atoms were placed in geometrically idealized positions $(\mathrm{C}-\mathrm{H}=0.93 \AA)$ and constrained to ride on their parent atoms with $\mathrm{U}_{\text {iso }}(\mathrm{H})=1.2$ (aromatic). All non-hydrogen atoms were refined anisotropically in $F^{2}$ mode. Crystal data, data collection and refinement details 40 for $\mathbf{I}_{\mathbf{B}}, \mathbf{I}_{\mathbf{B}(\mathbf{R T})}, \mathbf{I I}_{\mathbf{G}}$ and $\mathbf{I I I}_{\mathbf{Y}}$ are listed in Table S1. The structures were deposited at the Cambridge Data Centre and allocated with CCDC 942202-942204 and 980610 numbers.

\section{ACKNOWLEDGMENT}

We gratefully acknowledge the support from the MTA Lendület ${ }_{45}$ Programme. The authors are indebted to Professor Julius Vancso (University of Twente) for his continuous interest and support. We thank Márta Rockov for EA measurements and István Sajó for recording the PXRD spectra.

\section{Notes and references}

$50{ }^{a}$ Hungarian Academy of Sciences, MTA TTK SZKI Lendület Supramolecular Chemistry Laboratory, ${ }^{b}$ MTA TTK MFI and ${ }^{c}$ MTA TTK SZKI Laboratory of Theoretical Chemistry 1117 Budapest, Magyar Tudósok körútja 2, Hungary. E-mail: deak.andrea@ttk.mta.hu

$\dagger$ Electronic Supplementary Information (ESI) available: experimental 55 details, crystallographic and spectroscopic data.
1 Y. Sagara, T. Kato, Nat. Chem., 2009, 1, 605.

2 A. L. Balch, Angew. Chem. Int. Ed., 2009, 48, 2641.

3 H. Nakayama, J.-i. Nishida, N. Takada, H. Sato, Y. Yamashita, Chem. Mater., 2012, 24, 671.

4 X. Zhang, Z. Chi, Y. Zhang, S. Liu, J. Xu, J. Mater. Chem. C., 2013, 1, 3376.

5 C. G. Schäfer, M. Gallei, J. T. Zahn, J. Engelhardt, G. P. Hellmann, M. Rehahn, Chem. Mater., 2013, 25, 2309.

656 T. Mutai, H. Satou, K. Araki, Nat. Mater., 2005, 4, 685.

7 H. Zhang, Z. Zhang, K. Ye, J. Zhang, Y. Wang, Adv. Mater., 2006, 18, 2369.

8 D. Yan, D. G. Evans, Mater. Horiz., 2013, 1, 46.

9 Y. Dong, B. Xu, J. Zhang, X. Tan, L. Wang, J. Chen, H. Lv, S. Wen, B.

70 Li, L. Ye, B. Zou, W. Tian, Angew. Chem. Int. Ed., 2012, 51, 10782.

10 G. Zhang, J. Lu, M. Sabat, C. L. Fraser, J. Am. Chem. Soc., 2010, 132, 2160.

11 S. Perruchas, X. F. Le Goff, S. Maron, I. Maurin, F. Guillen, A. Garcia, T. Gacoin, J.-P. Boilot, J. Am. Chem. Soc., 2010, 132, 10967.

7512 W. Z. Yuan, Y. Tan, Y. Gong, P. Lu, J. W. Y. Lam, X. Y. Shen, C. Feng, H. H.-Y. Sung, Y. Lu, I. D. Williams, J. Z. Sun, Y. Zhang, B. Z. Tang, Adv. Mater., 2013, 25, 2837.

13 J. Zhou, Z. Chang, Y. Jiang, B. He, M. Du, P. Lu, Y. Hong, H. S. Kwok, A. Qin, H. Qiu, Z. Zhao, B. Z. Tang, Chem. Commun., 2013, 49, 2491.

14 J. Shi, N. Chang, C. Li, J. Mei, C. Deng, X. Luo, Z. Liu, Z. Bo, Y. Q. Dong, B. Z. Tang, Chem. Commun., 2012, 48, 10675.

15 Z. Chi, X. Zhang, B. Xu, X. Zhou, C. Ma, Y. Zhang, S. Liu, J. Xu, Chem. Soc. Rev., 2012, 41, 3878.

8516 S.-J. Yoon, J. W. Chung, J. Gierschner, K. S. Kim, M.-G. Choi, D. Kim, S. Y. Park, J. Am. Chem. Soc., 2010, 132, 13675.

17 X. Luo, J. Li, C. Li, L. Heng, Y. Q. Dong, Z. Liu, Z. Bo, B. Z. Tang, Adv. Mater., 2011, 23, 3261.

18 J. Wang, J. Mei, R. Hu, J. Z. Sun, A. Qin, B. Z. Tang, J. Am. Chem. Soc., 2012, 134, 9956.

19 Y. Hong, J. W. Y. Lam, B. Z. Tang, Chem. Commun., 2009, 4332.

20 Y. Liu, S. Chen, J. W. Y. Lam, P. Lu, R. T. K. Kwok, F. Mahtab, H. S. Kwok, B. Z. Tang, Chem. Mater., 2011, 23, 2536.

21 W. Z. Yuan, Y. Gong, S. Chen, X. Y. Shen, J. W. Y. Lam, P. Lu, Y.

95 Lu, Z. Wang, R. Hu, N. Xie, H. S. Kwok, Y. Zhang, J. Z. Sun, B. Z. Tang, Chem. Mater., 2012, 24, 1518.

22 M Saitoh, A. L. Balch, J. Yuasa, T. Kawai, Inorg. Chem., 2010, 49, 7129.

23 Y.-A. Lee, J. E. McGarrah, R. J. Lachicotte, R. Eisenberg, J. Am. 100 Chem. Soc., 2002, 124, 10662.

24 L. Hao, R. J. Lachicotte, H. J. Grysling, R. Eisenberg, Inorg. Chem., $1999, \mathbf{3 8}, 4616$.

25 S. H. Lim, M. M. Olmstead, A. L. Balch, J. Am. Chem. Soc., 2011, 133, 10229.

10526 R. L. White-Morris, M. M. Olmstead, A. L. Balch, J. Am. Chem. Soc., 2003, 125, 1033.

27 M. A. Malwitz, S. H. Lim, R. L. White-Morris, D. M. Pham, M. M. Olmstead, A. L. Balch, J. Am. Chem. Soc., 2012, 134, 10885.

28 R. L. White-Morris, M. M. Olmstead, S. Attar, A. L. Balch, Inorg. 110 Chem., 2005, 44, 5021.

29 W. Lu, N. Zhu, C.-M. Che, J. Am. Chem. Soc., 2003, 125, 16081.

30 A. L. Balch, Gold Bulletin, 2004, 37, 45.

31 D. V. Toronto, B. Weissbart, D. S. Tinti, A. L. Balch, Inorg. Chem. 1996, 35, 2484-2489.

11532 E. J. Fernández, J. M. López-de-Luzuriaga, M. Monge, M. E. Olmos, J. Pérez, A. Laguna, A. A. Mohamed, J. P. Fackler, J. Am. Chem. Soc., 2003, 125, 2022

33 A. Laguna, T. Lasanta, J. M. López-de-Luzuriaga; M. Monge, P. Naumov, M. E. Olmos, J. Am. Chem. Soc., 2010, 132, 456.

12034 C. E. Strasser, V. J. Catalano, J. Am. Chem. Soc., 2010, 132, 10009.

35 J. C. Vickery, M. M. Olmstead, E. Y. Fung, A. L. Balch, Angew. Chem. Int. Ed., 1997, 36, 1179.

36 Z. Assefa, M. A. Omary, B. G. McBurnett, A. A. Mohamed, H. H Patterson, R. J. Staples, J. P. Fackler, Inorg. Chem., 2002, 41, 6274.

12537 V. C. Catalan, S. J. Horner, Inorg. Chem., 2003, 42, 8430. 
38 A. M. Kuchison, M. O. Wolf, B. O. Patrick, Chem. Commun., 2009, 7387.

39 I. O. Koshevoy, C.-L. Lin, A. J. Karttunen, M. Haukka, C.-W. Shih, P.-T. Chou, S. P. Tunik, T. A. Pakkanen, Chem. Commun., 2011, 47, 5533.

40 Y. A. Lee, R. Eisenberg, J. Am. Chem. Soc., 2003, 125, 7778.

41 J. Schneider, Y.-A. Lee, J. Pérez, W. W. Brennessel, C. Flaschenriem, R. Eisenberg, Inorg. Chem., 2008, 47, 957.

42 H. Ito, T. Saito, N. Oshima, N. Kitamura, S. Ishizaka, Y. Hinatsu, M.

10 Wakeshima, M. Kato, K. Tsuge, M. Sawamura, J. Am. Chem. Soc., 2008, 130, 10044.

43 M. Osawa, I. Kawata, S. Igawa, M. Hoshino, T. Fukunaga, D. Hashizume, Chem. Eur. J., 2010, 16, 12114.

44 H. Ito, M. Muromoto, S. Kurenuma, S. Ishizaka, N. Kitamura, H. Sato,

15 T. Seki, 2009, 4, DOI: 10.1038/ncomms3009.

45 C. Piguet, G. Bernardinelli, G. Hopfgartner, Chem. Rev., 1997, 97, 2005.

46 A. Deák, T. Megyes, G. Tárkányi, P. Király, L. Biczók, G. Pálinkás, P. J. Stang, J. Am. Chem. Soc., 2006, 128, 12668.

2047 G. Tárkányi, P. Király, G. Pálinkás, A. Deák, Magn. Reson. Chem., 2007, 45, 917.

48 T. Tunyogi, A. Deák, G. Tárkányi, P. Király, G. Pálinkás, Inorg. Chem., 2008, 47, 2049.

49 A. Deák, T. Tunyogi, Z. Károly, Sz. Klébert, G. Pálinkás, J. Am. Chem. Soc., 2010, 132, 13627.

50 W. Schuh, H. Kopacka, K. Wurst, P. Peringer, Chem. Commun., 2001, 2186.

51 D. Das, L. J. Barbour, Chem. Commun., 2008, 5110.

52 A. L. Balch, Gold Bulletin 2004, 37, 45.

3053 I. Mayer, Chem. Phys. Lett., 1983, 97, 270.

54 G. Kaupp, CrystEngComm, 2003, 5, 117.

55 D. E. Braun, V. Kahlenberg, T. Gelbrich, J. Ludescher, U. J. Griesser, CrystEngComm, 2008, 10, 1617.

56 A. Jayasankar, A. Somwangthanaroj, Z. J. Shao, N. Rodríguez-

35 Hornedo, Pharmaceutical Research, 2006, 23, 2381.

57 S. Raoux, W. Wełnik, D. Ielmini, Chem. Rev., 2010, 110, 240.

58 Ch. G. Chandaluri, T. P. Radhakrishnan, Angew. Chem. Int. Ed., 2012, 51, 11849.

59 A. Altomare, G. Cascarano, C. Giacovazzo, A. Guagliardi, M. C.

40 Burla, G. Polidori, M. J. Camalli, Appl. Cryst., 1994, 27, 435.

60 G. M. Sheldrick, Acta Crystallogr., 2008, A64, 112.

61 L. J. J. Farrugia, Appl. Crystallogr., 1999, 32, 837. 